\title{
Integrated optical devices design by genetic algorithm
}

\author{
L. Sanchis, A. Håkansson, D. López-Zanón, J. Bravo-Abad, ${ }^{a)}$ and José Sánchez-Dehesa ${ }^{\text {b) }}$ \\ Centro de Tecnología Nanofotonica, ETSI Telecomunicación, Universidad Politécnica de Valencia, \\ c/Camino de Vera s/n, E-46022 Valencia, Spain
}

(Received 15 October 2003; accepted 22 March 2004; published online 14 May 2004)

\begin{abstract}
In this work, we use multiple scattering in conjunction with a genetic algorithm to reliably determine the optimized photonic-crystal-based structure able to perform a specific optical task. The genetic algorithm operates on a population of candidate structures to produce new candidates with better performance in an iterative process. The potential of this approach is illustrated by designing a spot size converter that has a very low $F$ number $(F=0.47)$ and a conversion ratio of 11:1. Also, we have designed a coupler device that introduces the light from the optical fiber into a photonic-crystal-based waveguide with a coupling efficiency over $87 \%$ for a wavelength that can be tuned to $1.5 \mu \mathrm{m}$. (C) 2004 American Institute of Physics. [DOI: 10.1063/1.1738931]
\end{abstract}

A new generation of optical devices is envisaged thanks to the properties of photonic crystals (PCs). ${ }^{1}$ In recent years much attention has been focused on systems based on twodimensional (2D) PCs because of their easiness in the fabrication process. Thus, very compact optical devices and circuits can be designed by introducing point and/or line defects. In order to use such PC circuits in actual applications it is necessary to establish a connection with an optical fiber. However, the core of the optical fiber is about one order of magnitude larger than the PC-based waveguide. Therefore, the design of an efficient (low loss) spot size converter is a crucial goal in the field of PC; its solution will introduce the PC devices in the marketplace. In this regard, several groups ${ }^{2-7}$ have tackled this problem by using different approaches. Most of them proposed tapered waveguide structures, ${ }^{2-5}$ or by using reflective structures to focus the light into the waveguide. ${ }^{6}$ A different approach consists of using the anisotropy of the PCs equifrequency surfaces. ${ }^{7}$

This letter introduces a method that is useful in determining the optimized configuration of a 2D-PC structure capable of performing a requested optical task with high efficiency. The method is illustrated by finding a spot size converter (lens) that has a conversion ratio 11:1. In addition, the designed PC structure that involves a spot-size converter in connection with a PC-based waveguide is presented. The insertion loss predicted for this new structure is about $13 \%$, which is of the lowest reported by numerical simulations based on different coupling mechanisms. ${ }^{2-6}$

Our method is based on a binary-coded genetic algorithm (GA), an optimization strategy inspired by Darwinian evolution. ${ }^{8}$ We consider an hexagonal lattice of Si dielectric cylinders $\left(n_{\mathrm{Si}} \approx 3.46\right.$ at $\left.1.5 \mu \mathrm{m}\right)$ in a background of silica $\left(n_{\mathrm{SiO}_{2}}=1.45\right)$. We employ the multiple scattering theory (MST) to study the diffraction effects of the TM modes (inplane magnetic field). The MST has been successfully ap-

\footnotetext{
${ }^{a}$ Permanent address: Departamento de Física Teórica de la Materia Condensada, Facultad de Ciencias (C-V), Universidad Autónoma de Madrid, E-28049 Madrid, Spain.

b)Electronic mail: jsdehesa@upvnet.upv.es
}

plied in the analysis of metallic and dielectric clusters based on 2D PCs. ${ }^{9}, 10$

As the first step in the process, a structure made of a fixed number of cylinders is chosen. The GA is then implemented with the possibility of removing cylinders from the cluster. A string of binary digits represents a possible structure (individual). Each binary digit (1 means presence and 0 absence of a cylinder at a given position) is called a gene. Each individual is associated with a value (the fitness), which is strictly related to the PC property that we are looking for. A crystal with a well asked for property will have a high fitness and vice versa. A certain number of individuals (population) is created by the GA that randomly determines their genes.

After the initiation, the GA selects two individuals at random, the one with the highest fitness acts as a parent, and the second parent chosen in the same way. Presently, a uniform-crossover operator randomly exchanges the chromosomes of the two parents with probability $C$ and, in this way, an offspring is created. This is done until we have a new equal sized population of offspring, i.e., a second generation. The selection and crossover operators tend to enable the evolutionary process to move toward promising regions of the search space. Then, a mutation operator is introduced to prevent premature convergence to local maxima. This operator changes the value of the genes at random with a probability $\mu$. The three operators are repeatedly applied to the population, with the constrain that the best-fitted individual is always copied into the next generation (elitism). As there is no way to know if the global maximum is reached, this process is iterated until there is no improvement of the fitness value of the best individual in the population. For further details see Holland ${ }^{8}$ and Goldberg. ${ }^{11}$

The fitness parameter in our calculations is equal to the component of the Poynting vector, $\mathbf{S}$, along the propagation direction of the incident beam, which is evaluated in a selected position or in a set of positions depending on the desired result. The Poynting vector is calculated by the MST. The external radiation field is a Gaussian beam in order to demonstrate the spot-size conversion. The beam is formed as a sum of plane waves weighted with a function dependent of 
the beam waist radius $w_{0}$. This beam represents the light at the output of a fiber, its diameter $2 w_{0}$ being the diameter of the fiber core (about $8-10 \mu \mathrm{m}$ ).

We now deal with the design of the spot-size converter. We assume that a TM polarized Gaussian beam (centered at the origin of our coordinate system) propagates along the $x$ axis. This beam impinges on a crystal consisting of a hexagonal array of Si cylinders in silica, the $z$ axis being parallel to the cylinder axis. The size of this crystal along the $y$ direction must be chosen slightly larger than the incidentbeam width to avoid flux escaping at the lateral borders of the crystal, hence we have chosen a crystal made of 26 rows of cylinders whose total length is about $3 w_{0}$ along the $y$ axis. Regarding its thickness along the $x$ axis, we used 13 columns of cylinders. The total number of cylinders is 318 , which is conditioned by our calculation resources. This structure constitutes the lens material (LM) of our integrated device. We consider a lattice constant $a_{\mathrm{LM}}$ and radius $r=0.294 a_{\mathrm{LM}}$. A band structure calculation of the corresponding infinite crystal by means of a plane wave expansion method predicts a forbidden gap for the TM-like modes in the range of frequencies $0.222-0.292$ (in units of $2 \pi c / a_{\mathrm{LM}}$ ). In order to have a low reflectance device we choose a working frequency of 0.197 , i.e., below the first gap, where propagation is allowed in all directions. On the other hand, the condition imposed to the GA in order to produce the spot-size conversion is to maximize the $x$ component of the Poynting vector $S_{x}$ at one point (the focus) behind the cylinders. A full account of how the MST is used to get this magnitude can be found in Ref. 10 and references therein. The CPU time needed to get the fitness of one individual is of the order of $6 \mathrm{~s}$ in a $2.8 \mathrm{GHz}$ Pentium IV workstation. The coordinates of the focal point $\left(x_{f}, y_{f}\right)$ are freely determined. Particularly, we impose the focal point to be located on the symmetry axis of the system $\left(y_{f}=0\right)$, and with an $x$ coordinate $x_{f}=4.60 \lambda_{0}$, where $\lambda_{0}$ is fixed by the working frequency $\left(\lambda_{0} \equiv a_{\mathrm{LM}} / 0.197\right)$. Our results indicate that under this simple condition the losses due to reflectance diminish and, at the same time, considerably reduces the waist of the propagated beam at the focus.

We realize that the global maximum must correspond to a symmetric structure, which is obvious from the symmetry of the problem. Then, the GA is forced to deal only with symmetric configurations. Therefore, the space search size is reduced from $2^{N}$ to $2^{(N+n) / 2}$ possible configurations, with $N=318$ and $n=6$, where $N$ is the number of cylinders of the LM, and $n$ the number of cylinders centered on the symmetry axis from $5.3 \times 10^{95}$ possible configurations to 5.8 $\times 10^{48}$. We have employed a crossover operator with a probability $C=0.4$, which is maintained in the calculations, and a population $P=400$ individuals. The left-hand side panel in Fig. 1 shows the resulting spot-size converter device and the pattern of the corresponding electric field modulus. Two different scales are used to measure distances, lattice parameter units (bottom and left) and wavelength units (top and right). The spot-size conversion can clearly be observed in the right-hand side panel of Fig. 1, where $S_{x}$ is represented along the direction parallel to the $y$ axis at the $x$ coordinate of the focal point (thin line), and for the incident beam at the $y$ axis $(x=0)$ (thick line). A fit of $S_{x}\left(x_{f}, y\right)$ to a Gaussian along the $y$ direction gives a beam waist radius of $w_{0}^{\prime}=0.30 \lambda_{0}$. This Downloaded 09 Jun 2004 to 158.42.89.222. Redistribution subject

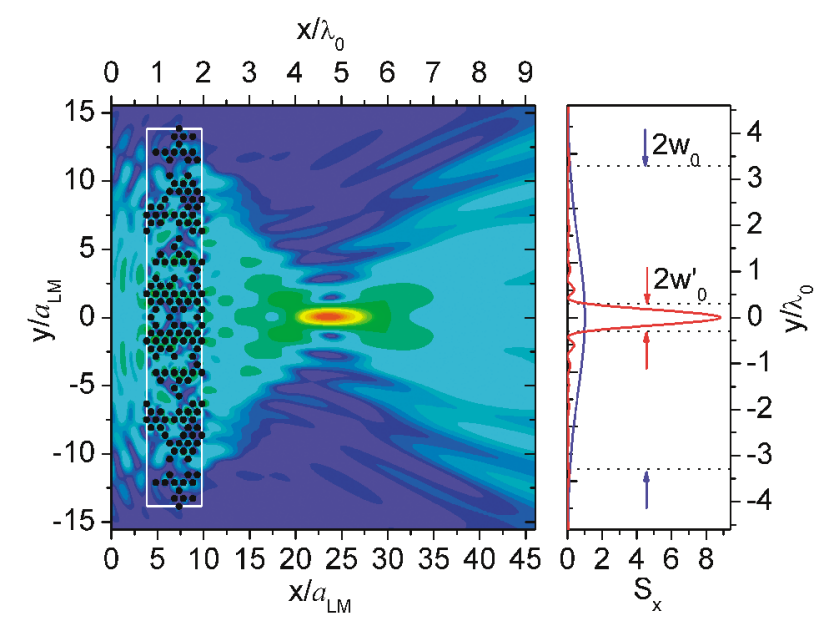

FIG. 1. (Color) (Left inset) Focusing effect produced by a spot-size converter (lens) based on a 2D photonic crystal (black dots). The pattern of the electric field modulus is represented in a wide spatial region. The length scales are given in terms of the lattice parameter employed in the design of the lens, $a_{\mathrm{LM}}$, as wells as in terms of the working wavelength of the lens $\lambda_{0}$. The white triangle defines the region where the cylinders' positions have been optimized by GA. (Right inset) A scale color is used, red (blue) color means maximum (minimum) electric field modulus. The $x$-component of the Poynting vector represented at the focal point $\left(x_{f}=4.60 \lambda_{0}\right)$.

means that the spot-size converter has a conversion ratio of $3.30 \lambda_{0}: 0.30 \lambda_{0} \approx 11: 1$, sufficient for classical-PC waveguide mode coupling requirements. In addition, this lenstype structure shows another property that is difficult to achieve by conventional lens design, its low $F$ number. According to Gaussian optics $F=(2 \pi) w_{0}^{\prime} / 4 \lambda_{0}$, which in our lens structure takes a value of only 0.47 . On the other hand, the power loss, which is defined as the ratio of the transmitted power calculated inside the focus waist $\left(2 w_{0}^{\prime}\right)$ to that of the incident beam, takes the value of $1.0 \mathrm{~dB}$. In other terms, $79 \%$ of the incident power is squeezed and passes through the focus. At this point Kosaka et al. ${ }^{7}$ reported a photoniccrystal spot-size converter that reduces the spot-size in the ratio 10:1, but no information is provided on the power passing through the focus.

We now discuss the problem of designing an integrated device involving the coupling of the light squeezed by the spot-size converted into the PC waveguide. The parameters of the PC waveguide, [which we name guide material (GM)], must be chosen carefully in order to allow the coupling with a state localized in the waveguide. First, the photonic band structure must have a full gap at the working frequency of the LM and second, a guided mode of the crystal with a missing row of cylinders, must exist at such a frequency. Thus, we choose the lattice parameter of the GM, $a_{\mathrm{GM}}$ $=1.5 a_{\mathrm{LM}}$. On the other hand, we keep the same orientation and cylinder radius as in the LM, and the PC waveguide is created along the $\Gamma K$ direction in the hexagonal lattice.

If one simply places the entry of the waveguide at the focal point of the lens, one obtains a total insertion loss as high as $7.03 \mathrm{~dB}$. This efficiency is calculated as the ratio of the total power that is transmitted through the waveguide to the incident power. The optimization process begins at this point by defining a set of 52 cylinders, which are placed in front of the entrance in order to facilitate the coupling to the waveguide mode, and letting the GA operate over it. These cylinders have the same symmetry, lattice parameter, and to AlP license or copyright, see http://apl.aip.org/apl/copyright.jsp 


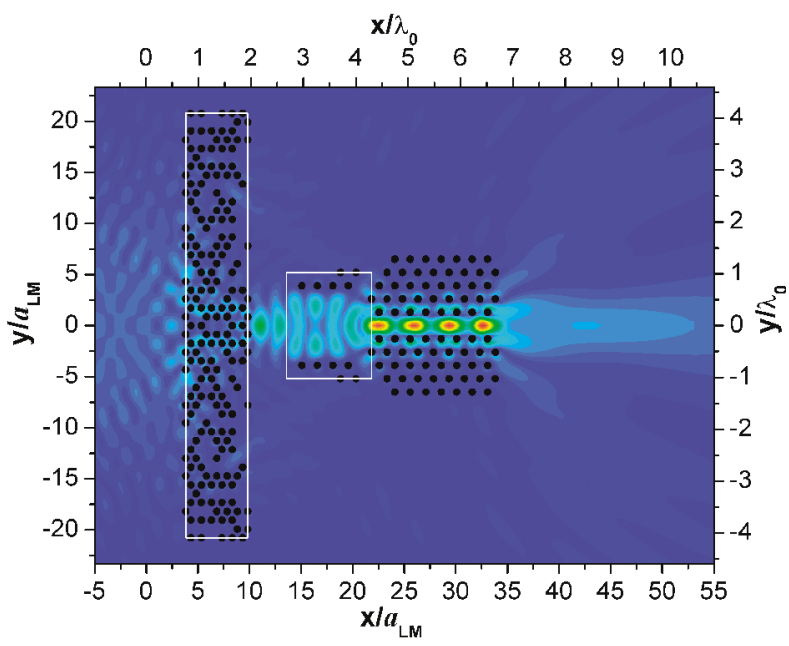

FIG. 2. (Color) Optimized waveguided-coupler device (dots) obtained by a genetic algorithm. The white rectangles enclosed the cylinders where the GA is applied. A scale color is used; red (blue) color defines the maximum (minimum) electric field modulus.

orientation as the GM. The fitness was set equal to the sum of $S_{x}$ calculated at 30 points located along a transversal segment defined at the end of the waveguide; i.e., on the segment $\left[-5.2 a_{\mathrm{LM}},+5.2 a_{\mathrm{LM}}\right]$. We allow the GA to act over the full structure made of the lens and the mouth. Now, it contains $162+32$ genes and, consequently, the space search size has been increased up to $2^{162+32} \simeq 2.5 \times 10^{58}$. In this case we consider that each generation contains a population of 600 individuals. Figure 2 plots the optimized integrated device (the waveguide coupler) together with its electric field modulus pattern. The insertion loss predicted for this structure is as low as $0.61 \mathrm{~dB}$. This means that $87 \%$ of the impinging light passes through the waveguide and is detected at the output. In fact, this value is underestimated since it does not include the light reflected at the end of the waveguide by finite size effect. Therefore, the coupling efficiency predicted by this new structure is comparable with the ones reported in the literature. Thus, a waveguide-to-fiber coupling improvement exceeding $2 \mathrm{~dB}$ per converter is shown in Ref. 2. References 3 and 4 reported two different tapered couplers that numerically have over $90 \%$ power transmission. The simulations by Sanchis et al. ${ }^{5}$ reported that a transmission over $84 \%$ can be achieved if defects are put on a planar photonic crystal tapered waveguide. Finally, the $J$ coupler proposed by Prather et al. ${ }^{6}$ predicts a coupling efficiency of $91 \%$. In spite of the large coupling efficiencies reported for these devices, in comparison with the one designed here, the low compactness and integrability of these devices are their main drawbacks when they are compared with the one designed by evolutionary programming.

Although our simulations involved the simplifying assumption of 2D PCs, it should be noticed that such 2Dperiodic crystals can be studied in actual 3D crystals. For example, our recent simulations of $2 \mathrm{D}$ prism ${ }^{10}$ reproduces fairly well the behavior of the actual structures in the microwave regime. We conjecture that similar results also would be obtainable in the optical regime by using a PC slab sandwiched between multilayers films with a large gap. Also, the preceding discussion focused on the TM modes of a structure based on "dielectric-scatterers-on-background." However, with this general method presented here, similar devices based on "holes-in-dielectric" structures can also be designed.

This work was supported in part by the Spanish CICYT (Project No. MAT2000-1670-C04), CAM (Ref. 07N/0059/ 2002), and the EU under Contract No. PHOBOS-IST-199919009. We also acknowledge the computing facilities provided by the Centro de Computación Científica at the UAM.

${ }^{1}$ J. D. Joannopoulos, R. D. Mead, and J. N. Winn, Photonic Crystals (Princeton University Press, Princeton, NJ, 1995).

${ }^{2}$ M. M. Spühler, B. J. Offrein, G.-L Bona, R. Germann, I. Massarek, and D. Erni, J. Lightwave Technol. 16, 1680 (1998).

${ }^{3}$ A. Mekis and J. D. Joannopoulos, J. Lightwave Technol. 19, 861 (2001)

${ }^{4}$ W. Kuang, C. Kim, A. Stapleton, and J. D. O'Brien, Opt. Lett. 27, 1605 (2002).

${ }^{5}$ P. Sanchis, J. Martí, A. García, A. Martínez, and J. Blasco, Electron. Lett. 38, 961 (2002).

${ }^{6}$ D. W. Prather, J. Murakowski, S. Shi, S. Venkataraman, A. Sharkawy, C. Chen, and D. Pustai, Opt. Lett. 27, 1601 (2002).

${ }^{7}$ H. Kosaka, T. Kawashima, A. Tomita, T. Sato, and S. Kawakami, Appl. Phys. Lett. 76, 268 (2000).

${ }^{8}$ J. H. Holland, Adaptation in Natural and Artificial Systems (The University of Michigan Press, Ann Arbor, 1975).

${ }^{9}$ T. Ochiai and J. Sánchez-Dehesa, Phys. Rev. B 65, 245111 (2002).

${ }^{10}$ J. Bravo-Abad, T. Ochiai, and J. Sánchez-Dehesa, Phys. Rev. B 67, 115116 (2003).

${ }^{11}$ D. E. Goldberg, Genetic Algorithms in Search, Optimization and Learning (Addison, Reading, MA, 1989). 